\title{
Prevalence of retinal changes in patients with systemic sclerosis: the association between retinal vascular changes and nailfold capillaroscopic findings
}

\author{
Saeedeh Shenavandeh ${ }^{1}$ ID , Mehrdad Afarid $^{2}$ ID , Tarlaan Hasanaghaei $^{1}$ ID , \\ Mohammad Ali Nazarinia1,3 ID \\ ${ }^{1}$ Shiraz University of Medical Sciences, Department of Internal Medicine, Division of Rheumatology, Namazee Hospital, Shiraz, Iran \\ ${ }^{2}$ Shiraz University of Medical Sciences, Departments of Ophthalmology, Poostchi Ophthalmology Research Center, Shiraz, Iran \\ ${ }^{3}$ Shiraz Geriatric Research Center, Shiraz University of Medical Sciences, Iran
}

\begin{abstract}
Background: Systemic sclerosis (SSc) is a connective tissue disease affecting the skin and multiple organs. Most of the evidence on ocular involvement comes from small studies. We evaluated the retinal vascular changes in patients with SSc and its associations with types, disease duration, skin score, and nailfold capillaroscopy changes.

Material and methods: We evaluated the demographic, clinical and nailfold capillaroscopy data of 52 patients referring to the clinic of scleroderma with SSc according to the 2013 ACR/EULAR SSC criteria. In addition, full ophthalmic examination in 52 patients and fundus photography in 40 patients were done in the ophthalmology clinic.

Results: There were 52 (49 women and 3 men, 17 diffuse and 35 limited) patients with SSc with mean disease duration of $8 \pm 5$ years and mean age of $40 \pm 9$ years. Retinal changes in the ophthalmologic examination were seen in $30.7 \%$ as increased vascular tortuosity. None of the patients had cotton wool spot, hemorrhage or hard exudate. Forty patients underwent fundus photography and $22.5 \%$ of them had vascular tortuosity. Only the presence of hemorrhage in the nailfolds was correlated with retinal tortuosity, and the other characteristics of nailfold capillaroscopy did not have any association with retinal changes.

Conclusions: Retinal vascular changes were seen in about one third of our patients. There was no correlation between nailfold capillaroscopy, disease duration, type and skin score of SSc patients and retinal vascular changes. These findings suggest that the mechanisms or the quality of changes in the retinal vessels and nailfold vasculature may be different.
\end{abstract}

Key words: systemic sclerosis, capillaroscopy, retinal tortuosity, nailfold capillaroscopy.

\section{Introduction}

Systemic sclerosis (SSc) is a generalized chronic connective tissue disorder of unknown etiology [1]. Diffuse fibroproliferative vasculopathy is one of the critical abnormalities in scleroderma most commonly involving the microvasculature system, leading to Raynaud's phenomenon and digital ischemia [2]. Nailfold capillaroscopy is a non-invasive method that is increasingly used in the evaluation of patients with Raynaud's phenomenon and scleroderma spectrum of diseases $[3,4]$.
Capillary changes in patients with developing or established SSc are drop-out of the capillaries, enlargement of the capillary loops, microhemorrhages, neoangiogenesis and changes in the capillary morphology and architecture [5]. There are multiple studies on the relationship of the severity of capillary findings and clinical manifestations and some poor prognostic manifestations of patients with SSc, such as pulmonary hypertension and digital ulcers [6-9].

Since systemic sclerosis is a rare disease, most of the data regarding ocular involvement come from single

Address for correspondence:

Tarlaan Hasanaghaei, Shiraz University of Medical Sciences, Department of Internal Medicine, Division of Rheumatology, Namazee Hospital, Shiraz, Iran, Po Box: 71345-1414, e-mail: tarlaanh@gmail.com, ORCID: https://orcid.org/0000-0003-0522-7186 Submitted: 26.11.2020; Accepted: 16.01.2021 
case reports or studies with a small number of samples [10-13]. Some of the reported features in SSc are well known, such as eyelid skin changes, dry eye and astigmatism, but others are not well understood, e.g. scleroderma retinopathy, although choroidal circulation disorders have been reported in more than 33\% of SSc patients in some studies, e.g. that of Waszczykowska et al. [14]. A recent review of the literature by Ushiyama et al. showed a prevalence of $34 \%$ for retinal changes [12].

In SSc, small vessels are mostly affected and, in some studies, cotton-wool spots in the retina, frequent atrophic lesions of the retinal pigment epithelium within the posterior pole of the retina, dilatation of the venous vessels, intra-retinal extravasation, hard exudates, and macular edema have been reported [10, 11, 14-16].

In a few studies, some evidence of the relationships between nailfold capillaroscopy and retinal changes has been reported. Gomes et al. [10] found that retinal microvascular abnormalities are indistinguishable from those related to systemic hypertension in patients with SSc and they are associated with severe capillaroscopic patterns; however, they did not find any relationship between eyelid skin changes, dry eye and capillaroscopy patterns in patients with SSc. In a study by Ushiyama et al., there was no relationship between retinal vascular changes and nailfold capillary damage [12].

In this study, we evaluated the retinal changes including vascular tortuosity, hemorrhage and hard exudates in patients with SSc using fundus photography and retinal examination by an ophthalmologist. In addition, we searched for any association between nailfold capillaroscopy and retinal vascular changes and aimed to determine whether we could use capillaroscopy as a predictor of retinal involvement in patients with SSc.

\section{Material and methods}

The authors included 52 adult patients with SSc who fulfilled the 2013 ACR/EULAR SSc diagnostic criteria [17]. They actively participated in the study and referred to the clinic of scleroderma of Hafez Hospital or were admitted to the Rheumatology Ward of Hafez Hospital affiliated to Shiraz University of Medical Sciences, in the period from January 2016 to January 2017, with an age range of 16-65 years.

The exclusion criteria were cases with overlap syndrome, diabetes mellitus, current smokers and patients with hypertension. In addition, patients with a history of previous ophthalmic surgery, any retinal pathology proven due to known diseases not related to scleroderma, intraocular inflammation, ocular hypertension, previous history of uncontrolled hypertension or renal crisis and a history of ocular trauma were excluded.
The sub-investigators received a checklist with inclusion and exclusion criteria. When a subject met the inclusion criteria while having no exclusion criteria, the data were registered.

The authors obtained the consent of the bioethical commission to conduct the present study.

All the patients signed a declaration of consent, before being included in the study population. All clinical manifestations of patients and their organ involvement were recorded during evaluation.

The rheumatologist involved in this research project performed the physical examination.

The demographic data including disease duration (date of first Raynaud's and non-Raynaud's symptoms); serological (anti-Scl-70, anti-centromere and antinuclear antibody), clinical [diffuse cutaneous SSc (dsSSc), and limited cutaneous SSc (IcSSc)] subsets according to LeRoy et al. [1]; and skin scoring according to the definition by LeRoy et al. in the MRSS (Modified Rodnan Score) [18] classified into 5 groups - normal, mild (1-14), moderate (15-29), severe (30-39) and end stage (40+) - were gathered. Pulmonary involvement was assessed by high resolution CT scan (HRCT) at the first screening or after detecting low diffusing capacity of lung for carbon monoxide (DLCO) $<80 \%$ or low forced expiratory volume $\left(\mathrm{FEV}_{1}\right)<75 \%$ in the pulmonary function test (PFT).

On the same day, the patients with SSc underwent capillaroscopy by stereomicroscope Euromax ST. 1740 at $250 \mathrm{~W}$ power and the video camera Cmex D.C. 5000 (5 megapixels). Immersion oil was applied on the nailfold bed to improve resolution. Eight fingers of the two hands excluding the thumbs were examined. We also took at least one image of the middle of nailfolds per finger, and the data were analyzed by a rheumatologist involved in this study.

The capillaroscopic data including capillary distribution (disturbed or normal), shape of capillaries (normal hairpin, subtle change including tortuosity and crossing, anomalies including meandering, ectasia, neo-angiogenesis and bizarre capillaries), the largest diameter of the arterial or venous side [dilated loops were defined as increase of capillary diameter $\geq 20$ and $<50 \mu \mathrm{m}$ ) and giant capillaries (a capillary with a homogeneously enlarged loop with a diameter $\geq 50 \mu \mathrm{m}$ )], capillary length (normal or elongated: $\geq 300 \mu \mathrm{m}$ ), mean capillary density (capillary loss defined as reduction of the normal number of capillaries below 7 per linear millimeter), avascular area (intercapillary distance $>500 \mu \mathrm{m}$ ): present or absent, microhemorrhages: present or absent, and neoangiogenesis or ramified capillaries: present or absent, were recorded in a form [19].

The whole findings were defined as normal, specific scleroderma changes for early, active, and late sclero- 
derma pattern, or non-specific abnormalities based on recommendation of Cutulo et al. [20].

ESR was measured by the Westergren method. Serum CRP by nephelometry; RF by latex test; ANA and anticentromere Ab or anti-Scl70 by the ELISA method were checked.

The patients who fulfilled the inclusion criteria were referred to the ophthalmology clinic of Poostchi to undergo noninvasive fundus color photography. The fundus photography was done using the fundus camera TRC 50 EX (Topcon) following pupil dilatation with 1\% tropicamide solution (eye drops) and then the patients were referred to Motahari clinic where retinal evaluation was done by a board certified and expert posterior segment ophthalmologist blinded to rheumatologic manifestations of patients. For each participant, a full ophthalmic examination, including best corrected visual acuity, slit-lamp examination, tonometry, and fundoscopy, was done. The important findings in the retinal examination were the existence of any exudate, hemorrhage and vascular morphological changes. Findings were reported qualitatively.

Statistical analysis was done using SPSS-19 and descriptive statistics and $\chi^{2}$ and $t$-test. The level of $p<0.05$ was considered significant.

\section{Results}

The authors included 52 patients in the study. The age range of the study population was 20 to 62 years (mean age: $40 \pm 9$ years). Among the study participants, 3 (5.8\%) were male and 49 (94.2\%) were female. Their mean disease duration was $8 \pm 5$ years. There were 35 patients with limited and 17 with diffuse scleroderma. The clinical, laboratory tests and other details of capillaroscopy findings are also presented in Table I.

In ophthalmologic examination by an ophthalmologist: visual acuity was below 0.8 in 9 (17\%) patients, lid thickness was reported in $3(5.7 \%)$ patients, dry eye was reported in 8 (15.3\%) patients, cup to disc ratio was below $50 \%$ in all patients, mean ocular pressure was 14.05 \pm 2.74 , and three (5\%) patients had cataract. The limbus, cornea, iris and anterior chamber were normal in all patients. Retinal vascular changes in ophthalmologic examination were seen in $30 \%$ of patients as increased vascular tortuosity (mainly in large vessels that could be seen in examination). Fundus photography that was checked by the same ophthalmologist in 40 patients revealed that $22.5 \%$ had vascular tortuosity ( 52 patients were examined by the ophthalmologist and 40 of them underwent photography of the fundus). None of the patients had cotton wool spot, hemorrhage or hard exudate in retinal examination.
Then, we compared the association of abnormal vascular finding defined as increased vascular tortuosity in the ophthalmologist examination and fundus photography with capillaroscopy components of the patients' nailfolds, disease duration, and skin score of patients, as shown in Table II. Figure 1 presents eye fundus photographs and simultaneous nailfold capillaroscopy changes.

The capillaroscopic findings had no association with retinal findings, except for capillary hemorrhage in capillaroscopy, which was related to vascular tortuosity reported in ophthalmoscopy ( $p=0.014)$.

Type of scleroderma, skin score and disease duration had no association with retinal findings ( $p=0.882$, 0.670 and 0.622 ), respectively.

\section{Discussion}

In this study, the authors evaluated the prevalence of retinal vascular changes such as vascular tortuosity in patients with scleroderma. We also compared the retinal changes with capillaroscopy findings, type of scleroderma, duration of the disease, and skin score.

In the study of complete eye examination, $17 \%$ of our patients had decreased visual acuity. In another study by Waszczykowska et al. [14] on patients with SSc, there was no significant difference between the patients and control population in visual acuity. In addition, we found a low frequency of increased lid thickness that was reported in $3(5.7 \%)$ patients and also dry eye that was seen in 8 (15.3\%) patients. Cup to disc ratio was normal in all of our patients and mean ocular pressure was $14.05 \pm 2.74$. Three (5\%) patients had cataract. The limbus, cornea, iris and anterior chamber were normal in all patients.

Compared to other studies which used the Schirmer test for detection of dry eye, our patients mostly had normal intraocular pressure and a lower percentage of dry eye, while other authors reported a rate of $49 \%$ for dry eye in their patients, $42.2 \%$ for cataract, and about $13 \%$ for glaucoma [10].

Our study focused mostly on retinal changes. We found retinal vascular changes presenting as increased microvascular tortuosity in about $30.7 \%$ and $22.5 \%$ of the study participants in our ophthalmology examination and fundus photography, respectively.

Ushiyama et al. [12] reported the prevalence of 34\% for retinal vascular abnormalities including hard exudate, vascular tortuosity and macular degeneration in their patients with SSc. Choroid vasculature seemed to be more involved than retinal circulation in the systematic review by Kreps et al. [15]. In another study, by Jakhar et al. [21], prevalence of retinal changes was $28.89 \%$.

The prevalence of retinal vascular changes is in line with the results of our study, indicating a high preva- 
Table I. Demographic, capillaroscopy, skin score, clinical manifestations and laboratory features of patients and their association with types of SSc

\begin{tabular}{|c|c|c|c|}
\hline Variable & $\begin{array}{l}\text { All patients } \\
(n=52)\end{array}$ & $\begin{array}{c}\text { dcSSc } \\
(n=17)\end{array}$ & $\begin{array}{c}\text { IcSSc } \\
(n=35)\end{array}$ \\
\hline Average age (years) & $40( \pm 9)$ & $42( \pm 9)$ & $34( \pm 7)$ \\
\hline \multicolumn{4}{|l|}{ Gender, $n(\%)$} \\
\hline Female & $49(94.2)$ & $15(88)$ & $34(97)$ \\
\hline Male & $3(5.8)$ & $2(12)$ & $1(3)$ \\
\hline \multicolumn{4}{|l|}{ Mean disease duration, $n(\%)$} \\
\hline$<5$ years & $12(23.1)$ & $6(35)$ & $6(17)$ \\
\hline$\geq 5$ years & $40(76.9)$ & $11(65)$ & $29(83)$ \\
\hline Mean Raynaud's duration (years) & 3.1 & 2.3 & 3.4 \\
\hline \multicolumn{4}{|l|}{ Capillaroscopy pattern } \\
\hline Nonspecific & 0 & 0 & 0 \\
\hline \multicolumn{4}{|l|}{ Specific } \\
\hline Early pattern, $n(\%)$ & $7(13.4)$ & $1(6)$ & $6(18)$ \\
\hline Active pattern, $n(\%)$ & $29(55.7)$ & $10(59)$ & $19(54)$ \\
\hline Late pattern, $n(\%)$ & $16(30.7)$ & $6(35)$ & $10(28)$ \\
\hline Disturbed capillary distribution, $n$ (\%) & $46(88.4)$ & $16(94.1)$ & $30(85.7)$ \\
\hline Subtle changes, $n(\%)$ & $5(9.6)$ & $2(11.7)$ & $3(8.5)$ \\
\hline Anomalies & $30(58)$ & $10(58.8)$ & $20(57.1)$ \\
\hline Dilatation & $47(90.3)$ & $15(88.2)$ & $32(91.4)$ \\
\hline Giant loop > 0.05 & $28(53.8)$ & $10(58.8)$ & $18(51.4)$ \\
\hline Elongation & $23(44.2)$ & $5(29.4)$ & $18(51.4)$ \\
\hline Capillary loss < 7/mm & $43(82.7)$ & $16(94.1)$ & $27(77.1)$ \\
\hline Avascular area & $25(48)$ & $8(47)$ & $17(48.6)$ \\
\hline \multicolumn{4}{|l|}{ Skin score $n /$ total (\%) } \\
\hline Normal & 0/52 (0) & 0/17 (0) & 0/35 (0) \\
\hline Mild & $34 / 52(65.4)$ & $6 / 17(35.3)$ & $28 / 35(80)$ \\
\hline Moderate & $17 / 52(32.7)$ & 10/17 (58.9) & $7 / 35(20)$ \\
\hline Severe & $0 / 52(0)$ & 0/17 (0) & $0 / 35(0)$ \\
\hline End stage & $0 / 52(0)$ & 0/17 (0) & 0/35 (0) \\
\hline Missing & $1 / 52(1.9)$ & $1 / 17(5.8)$ & 0/35 (0) \\
\hline Raynaud's requiring vasodilator & $48 / 52(92.3)$ & $16 / 17(94.2)$ & $32 / 35(91.4)$ \\
\hline Missing & $3 / 52(5.8)$ & $1 / 17(5.8)$ & $2 / 35(5.7)$ \\
\hline Digital pitting scar, n/total (\%) & $29 / 52(55.8)$ & 10/17 (58.8) & $19 / 35(54.3)$ \\
\hline Missing & $3 / 52(5.8)$ & 0/17 (0) & $3 / 35(8.6)$ \\
\hline Digital tip ulcer, n/total (\%) & 9/52 (17.3) & $3 / 17(17.7)$ & 6/35 (17.1) \\
\hline Missing & $7 / 52(13.5)$ & $4 / 17(23.5)$ & $3 / 35(8.6)$ \\
\hline Digital gangrene, $n /$ total (\%) & $0 / 52(0)$ & 0/17 (0) & $0 / 35(0)$ \\
\hline FTP limitation, $n /$ total (\%) & 4/52 (7.7) & $1 / 17(5.8)$ & 3/35 (8.6) \\
\hline Missing & $6 / 52(11.5)$ & 4/17 (23.5) & $2 / 35(5.7)$ \\
\hline GI disturbance, $n /$ total (\%) & $37 / 52(71.1)$ & 13/17 (76.4) & $24 / 35(68.5)$ \\
\hline Pulmonary hypertension, n/total (\%) & $11 / 52(21.2)$ & $6 / 17(35.3)$ & $5 / 35(14.3)$ \\
\hline Missing & $5 / 52(9.6)$ & $3 / 17(17.6)$ & $2 / 35(5.7)$ \\
\hline
\end{tabular}


Table I. Cont.

\begin{tabular}{|lccc|}
\hline Variable & $\begin{array}{c}\text { All patients } \\
(n=52)\end{array}$ & $\begin{array}{c}\text { dcSSc } \\
(n=17)\end{array}$ & $\begin{array}{c}\text { IcSSc } \\
(n=35)\end{array}$ \\
\hline \begin{tabular}{l} 
Low DLCO, $n$ /total (\%) \\
\hline Missing
\end{tabular} & $15 / 52(28.8)$ & $4 / 17(23.5)$ & $11 / 35(31.4)$ \\
\hline Interstitial lung disease in HRCT, $n$ /total (\%) & $7 / 52(13.5)$ & $5 / 17(29.4)$ & $2 / 35(5.7)$ \\
\hline Missing & $20 / 52(38.5)$ & $4 / 17(23.5)$ & $16 / 35(45.7)$ \\
\hline Heart disease, $n$ /total (\%) & $3 / 52(5.8)$ & $2 / 17(11.8)$ & $1 / 35(2.9)$ \\
\hline Renal involvement, $n$ /total (\%) & $0 / 52(0)$ & $0 / 17(0)$ & $0 / 35(0)$ \\
\hline Immunosuppressive medication, $n$ /total (\%) & $0 / 52(0)$ & $0 / 17(0)$ & $0 / 35(0)$ \\
\hline ESR, $n$ /total (\%) & $52 / 52(100)$ & $17 / 17(100)$ & $35 / 35(100)$ \\
\hline$\geq 15$ & & & $24 / 35(68.6)$ \\
\hline$<15$ & $32 / 52(61.5)$ & $8 / 17(47)$ & $11 / 35(31.4)$ \\
\hline CRP positive & $20 / 52(38.5)$ & $9 / 17(53)$ & $3 / 35(8.6)$ \\
\hline Missing & $4 / 52(7.7)$ & $1 / 17(5.8)$ & $5 / 35(14.3)$ \\
\hline ANA positive, $n$ /total (\%) & $6 / 52(11.5)$ & $1 / 17(5.8)$ & $31 / 35(88.6)$ \\
\hline Missing & $47 / 52(90.4)$ & $16 / 17(94.1)$ & $4 / 35(11.4)$ \\
\hline Anti-SCL70 positive, $n /$ total (\%) & $4 / 52(7.7)$ & $0 / 17(0)$ & $17 / 35(48.6)$ \\
\hline Missing & $31 / 52(59.6)$ & $14 / 17(82.4)$ & $7 / 35(20)$ \\
\hline Anti-centromere positive, $n /$ total & $9 / 52(17.3)$ & $2 / 17(11.8)$ & $3 / 35(8.6)$ \\
\hline Missing & $3 / 52(5.8)$ & $0 / 17(0)$ & $19 / 35(54.3)$ \\
\hline
\end{tabular}

SSc - systemic sclerosis, FTP - finger to palm, DLCO - diffusion capacity of carbon monoxide, CRP - C-reactive protein, dcSSC - diffuse cutaneous systemic sclerosis, IcSSc - limited cutaneous systemic sclerosis.

lence of retinal vascular changes among scleroderma patients. Ushiyama et al. [12] reported a prevalence of $8 \%$ in the control group. In a recent study performed by Szucs et al. [22] 58.9\% of the patients had retinal and choroid changes found in the photography of the fundus.

In addition to specific pathologic alterations in retinal vasculature in the scleroderma, other factors may play a role in retinal vascular changes in scleroderma patients. These patients have a high prevalence of hypertension and hypertension crisis. Ushiyama et al. [12] reported higher systolic blood pressure and age in the group of scleroderma patients in comparison to the control group. Szucs et al. [22] did not exclude hypertensive patients. In our study, we excluded patients with diabetes mellitus and smokers, and we did not have any patient with a history of hypertensive crisis. Therefore, these etiologies have no role in the prevalence of retinal vascular changes in our study.

Aissopou et al. [23] found no difference in retinal vasculature of patients with SSc in comparison to the control group. In this study, the participants were non-diabetics with no history of hypertension and antihypertensive treatments. They concluded that the changes in vasculature in patients with SSc may be different in mechanism and severity compared with the retina and other parts of the body. Hypothetically, these characteristics may provide sparing of the retina from the "fibrous" vasculopathy seen in other organs of the body [23].

In our study, we found no significant relationship between scleroderma type and retinal vascular changes consisting of increased tortuosity. Duration of the disease and skin score had no association with retinal changes (all $p>0.05$ ). Similar findings were reported by Ushiyama et al. [12].

These findings indicate that the mechanisms of changes in retinal vasculature and nailfold vasculature may be at least partly different. Therefore, the changes in retinal vasculature and nailfold vasculature cannot be used for prediction of each other's prevalence. In addition, the findings support the conclusion of Aissopou et al. [23] that fibroproliferative vasculopathy in patients with SSc may depend on specific cellular or soluble factors not present in the retinal environment.

Based on the results of our study, duration of the disease had no effects on venular tortuosity in gross evaluation by the ophthalmologist and fundus photography. Aissopou et al. [23] also found that retinal vascular involvement in scleroderma was unrelated to disease duration.

In this study, skin score was not significantly different in scleroderma patients with different involvement 
Table II. Association of capillaroscopy findings with retinal vascular changes in ophthalmologist examination and digital fundus photography

\begin{tabular}{|c|c|c|c|c|}
\hline \multirow[t]{2}{*}{ Rheumatologic Clinical Variable } & \multicolumn{2}{|c|}{$\begin{array}{c}\text { Retinal examination, } \\
\text { vascular tortuosity } \\
n(\%)\end{array}$} & \multicolumn{2}{|c|}{$\begin{array}{c}\text { Fundus photograph, } \\
\text { vascular tortuosityn } \\
n(\%)\end{array}$} \\
\hline & $n(\%)$ & $p$-value & $n(\%)$ & $p$-value \\
\hline \multicolumn{5}{|l|}{ Capillary pattern } \\
\hline Early scleroderma pattern (abnormal/total) & $1 / 7(14.3 \%)$ & \multirow[t]{3}{*}{0.317} & $0 / 5(0 \%)$ & \multirow[t]{3}{*}{0.472} \\
\hline Active scleroderma pattern (abnormal/total) & $8 / 29(27.6 \%)$ & & $6 / 25(24 \%)$ & \\
\hline Late scleroderma pattern (abnormal/total) & $7 / 16(43.8 \%)$ & & $2 / 10(20 \%)$ & \\
\hline Disturbed capillary distribution (abnormal/total [No]) & $13 / 46(28.3 \%)$ & 0.357 & $8 / 35(22.9 \%)$ & 0.563 \\
\hline Subtle changes (abnormal/total [Yes]) & $2 / 5(40 \%)$ & 0.637 & $1 / 4(25 \%)$ & 1.000 \\
\hline Anomalies (abnormal/total [Yes]) & $1 / 4(25 \%)$ & 1.000 & $1 / 4(25 \%)$ & 1.000 \\
\hline Dilatation (abnormal/total [Yes]) & $14 / 47(29.8 \%)$ & 0.637 & $7 / 37(18.9 \%)$ & 0.498 \\
\hline Giant loop (abnormal/total [Yes]) & $7 / 28(25 \%)$ & 0.378 & $2 / 23(8.7 \%)$ & 0.053 \\
\hline Elongation (abnormal/total [Yes]) & $5 / 23(21.7 \%)$ & 0.242 & $6 / 19(31.6 \%)$ & 0.120 \\
\hline Capillary loss (abnormal/total [Yes]) & $12 / 43(27.9 \%)$ & 0.431 & $7 / 32(21.9 \%)$ & 1.000 \\
\hline Avascular area (abnormal/total [Yes]) & $8 / 25(32.0 \%)$ & 1.000 & $5 / 20(25.0 \%)$ & 0.695 \\
\hline Hemorrhage (abnormal/total [Yes]) & 6/33 (18.2\%) & 0.014 & $6 / 24(25.0 \%)$ & 0.439 \\
\hline Neo-angiogenesis (abnormal/total [Yes]) & $5 / 14(35.7 \%)$ & 0.738 & $3 / 9(33.3 \%)$ & 0.348 \\
\hline \multicolumn{5}{|l|}{ Scleroderma type } \\
\hline Limited (abnormal/total) & $11 / 35(31.4 \%)$ & \multirow[t]{2}{*}{0.882} & $4 / 27(14.8 \%)$ & \multirow[t]{2}{*}{0.400} \\
\hline Diffuse (abnormal/total) & $5 / 17(29.4 \%)$ & & $4 / 13(30.7 \%)$ & \\
\hline \multicolumn{5}{|l|}{ Skin score } \\
\hline Mild < 15 (abnormal/total) & $10 / 34(29.4 \%)$ & \multirow[t]{2}{*}{0.670} & $4 / 27(14.8 \%)$ & \multirow[t]{2}{*}{0.654} \\
\hline Moderate 15-29 (abnormal/total) & $6 / 17(35.2 \%)$ & & $3 / 12(25 \%)$ & \\
\hline \multicolumn{5}{|l|}{ Disease duration } \\
\hline$\geq 5$ years (abnormal/total) & $13 / 40(32.5 \%)$ & \multirow[t]{2}{*}{0.622} & $6 / 29(20.7 \%)$ & \multirow[t]{2}{*}{1.000} \\
\hline$<5$ years (abnormal/total) & $3 / 12(25.0 \%)$ & & 2/11 (18.2\%) & \\
\hline
\end{tabular}

of retinal vasculature. Similar to our findings, no relationship between retinal vascular changes and the extent of skin involvement was reported by Aissopou et al. [23].

We found increased retinal vascular tortuosity in one third of our patients in examination of the eye by the ophthalmologist and $22.2 \%$ by fundus photography. We did not find any parallel correlation between retinal tortuosity and the nailfold capillary patterns, except for hemorrhage, and possibly it was based on different mechanisms and functions of microvasculature in these different areas of the body. It is known that the nailfolds have a high density of arteriovenous anastomoses, which have direct connections between the arterioles and venules with no nutritional function, but instead these anastomoses function as thermoregulators. These sites are distinct from other skin areas due to their specialized structures and functional features for thermoregulation [24].
A previous study by Shenavandeh et al. [25] on the correlation of ear involvement and capillaroscopy of SSc patients also did not show any correlation between nailfoldcapillary changes and ear involvement, so it seems the pathogenic mechanisms in organs such as the ear and eye are different from nailfold capillaries in these patients.

We did not use angiography of the retina, as we did not try to use any invasive tool in our asymptomatic patients; so, this was one of our study limitations. Retinal angiography is probably more accurate than gross physical examination and fundus photography. Our retinal study was based on the examination of the ophthalmologist and fundus photography that was seen grossly, and the diameters and characteristics of the vessels were not calculated in detail and only grossly interpreted. In addition, the other limitation of our study was the lack of comparison with the control group, which should be done in future studies. 

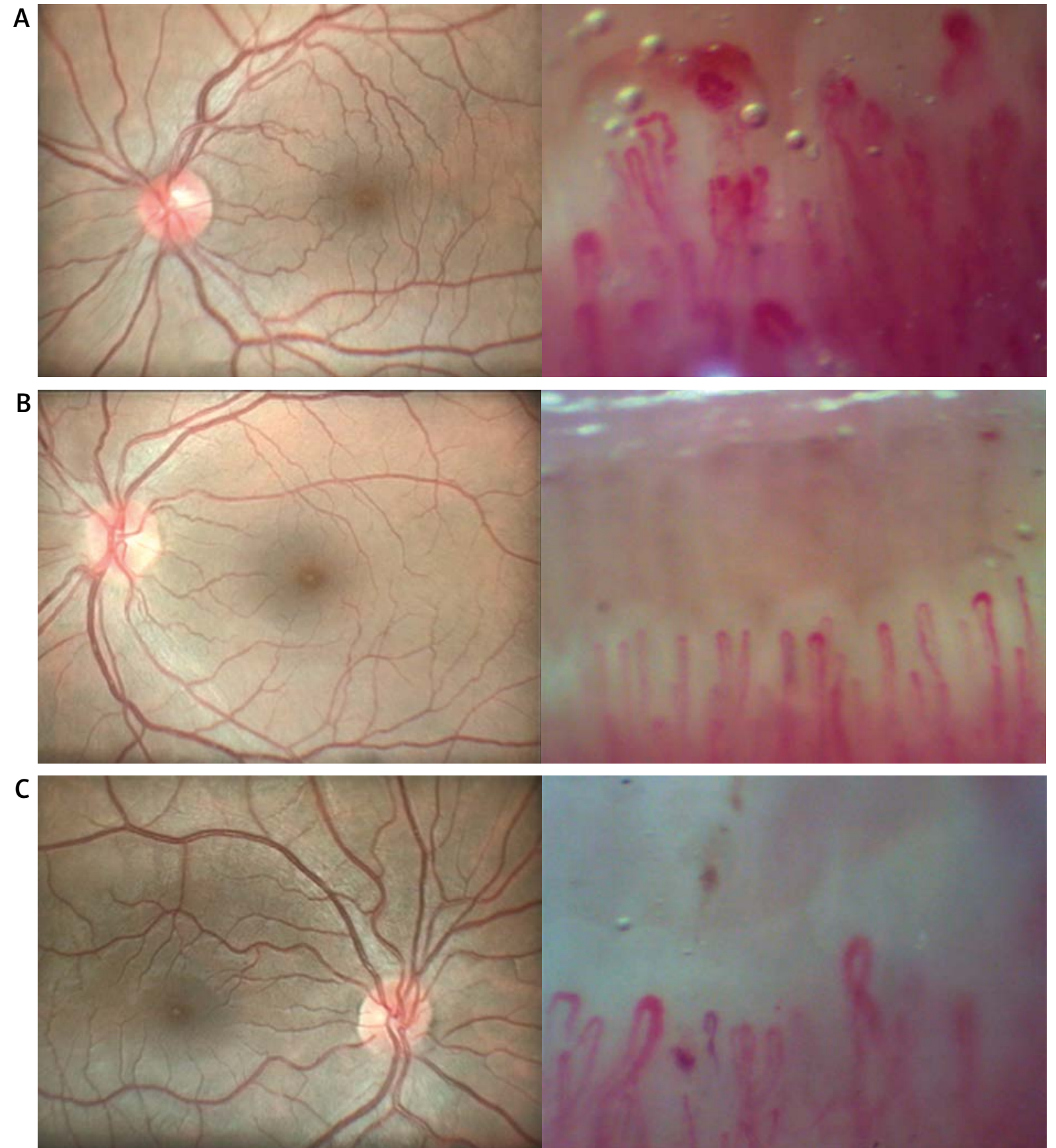

Fig. 1. Eye fundus photographs and simultaneous nailfold capillaroscopy changes. Normal fundus photography with increased tortuosity of the vessels and at the same time nailfold capillaroscopy that shows multiple homogeneously dilated capillaries, multiple microhemorrhages and severe disturbed distribution of capillaries (A), normal fundus photography and simultaneous multiple capillaries with apical dilatation and rare microhemorrhages (B), normal fundus photography and simultaneous multiple homogeneously dilated capillaries and a few microhemorrhages in capillaroscopy of nailfolds (C).

\section{Conclusions}

Retinal vascular changes defined as vascular tortuosity were observed in one third of scleroderma patients by ophthalmologist examination and slightly lower in the fundus photography. There was no significant difference in the retinal vascular changes and skin score, capillaroscopic characteristics, and disease duration, except for the patients who had hemorrhage in nailfold 
capillaries. These findings indicate that we need to conduct studies on pathologies in smaller vessels of the eye.

Although nailfold capillaroscopy at present cannot predict the retinal changes in scleroderma patients, advances in our retinal examination techniques to obtain information about smaller vascular structures will help us in the future to predict the development of retinal changes by nailfold capillaroscopy and instigate early treatment for retinal involvement.

\section{Acknowledgements}

The authors would like to thank Shiraz University of Medical Sciences, Shiraz, Iran and also the Center for Development of Clinical Research of Namazee Hospital and Dr. Nasrin Shokrpour for editorial assistance.

\section{Funding}

The present article was extracted from the thesis written by Dr. Tarlan Hasanaghaei supported by Shiraz University of Medical Science Grant number: 94-01-49-10306.

No other specific funding was received from any funding bodies in the public, commercial or not-for-profit sectors to carry out the work described in this manuscript.

The authors declare no conflicts of interest.

\section{References}

1. LeRoy EC, Black C, Fleischmajer R, et al. Scleroderma (systemic sclerosis): classification, subsets and pathogenesis. J Rheumatol 1988; 15: 202-205.

2. Strange G, Nash P. The manifestations of vasculopathy in systemic sclerosis and its evidence-based therapy. Int J Rheum Dis 2009; 12: 192-206, DOI: 10.1111/j.1756-185X.2009.01410.x.

3. Hofstee HM, Serné EH, Roberts C, et al. A multicentre study on the reliability of qualitative and quantitative nail-fold videocapillaroscopy assessment. Rheumatology (Oxford) 2012; 51: 749-755, DOI: 10.1093/rheumatology/ker403 .

4. Cutolo M, Sulli A, Pizzorni C, Accardo S. Nailfold videocapillaroscopy assessment of microvascular damage in systemic sclerosis. J Rheumatol 2000; 27: 155-160.

5. Cutolo M, Pizzorni C, Meroni M, et al. The role of nailfold videocapillaroscopy in Raynaud's phenomenon monitoring and early diagnosis of systemic sclerosis. Reumatismo 2010; 62: 237-247, DOI: 10.4081/reumatismo.2010.237.

6. Cutolo M, Herrick AL, Distler O, et al. Nailfold videocapillaroscopic features and other clinical risk factors for digital ulcers in systemic sclerosis: a multicenter, prospective cohort study. Arthritis Rheumatol 2016; 68: 2527-2539, DOI: 10.1002/art.39718.

7. Pizzorni C, Giampetruzzi AR, Mondino C, et al. Nailfold capillaroscopic parameters and skin telangiectasia patterns in patients with systemic sclerosis. Microvasc Res 2017; 111: 20-24, DOI: 10.1016/j.mvr.2016.12.003.

8. Sebastiani M, Manfredi A, Colaci M, et al. Capillaroscopic skin ulcer risk index: a new prognostic tool for digital skin ulcer development in systemic sclerosis patients. Arthritis Rheum 2009; 61: 688-694, DOI: 10.1002/art.24394.

9. Emrani Z, Karbalaie A, Fatemi A, et al. Capillary density: an important parameter in nailfold capillaroscopy. Microvasc Res 2017; 109: 7-18, DOI: 10.1016/j.mvr.2016.09.001.

10. Gomes B de AF, Santhiago MR, Magalhães P, et al. Ocular findings in patients with systemic sclerosis. Clinics (Sao Paulo) 2011; 66: 379-385, DOI: 10.1590/s1807-59322011000300003.

11. Ingegnoli F, Gualtierotti R, Pierro L, et al. Choroidal impairment and macular thinning in patients with systemic sclerosis: the acute study. Microvasc Res 2015; 97: 31-36, DOI: 10.1016/j.mvr. 2014.08.008.

12. Ushiyama O, Ushiyama K, Yamada T, et al. Retinal findings in systemic sclerosis: a comparison with nailfold capillaroscopic patterns. Ann Rheum Dis 2003; 62: 204-207, DOI: 10.1136/ ard.62.3.204.

13. Malik F, Al Habash A. Presentation of acute central retinal vein occlusion in scleroderma. Saudi J Ophthalmol 2015; 29: 156-159, DOI: 10.1016/j.sjopt.2014.09.014.

14. Waszczykowska A, Gos R, Waszczykowska E, et al. Prevalence of ocular manifestations in systemic sclerosis patients. Arch Med Sci 2013; 9: 1107-1113, DOI: 10.5114/aoms.2013.39217.

15. Kreps EO, Carton C, Cutolo M, et al. Ocular involvement in systemic sclerosis: a systematic literature review, it's not all scleroderma that meets the eye. Semin Arthritis Rheum 2019; 49: 119-125, DOI: 10.1016/j.semarthrit.2018.12.007.

16. Peter S, Dietrich H, Wick G. Investigations for retinopathy in an avian model for systemic sclerosis. Exp Eye Res 2004; 79: 85-92, DOI: 10.1016/j.exer.2004.02.012.

17. van den Hoogen F, Khanna D, Fransen J, et al. 2013 classification criteria for systemic sclerosis: an American College of Rheumatology/European League against Rheumatism collaborative initiative. Arthritis Rheum 2013; 65: 2737-2747, DOI: 10.1002/art.38098.

18. Clements PJ, Lachenbruch PA, Seibold JR, et al. Skin thickness score in systemic sclerosis: an assessment of interobserver variability in 3 independent studies. J Rheumatol 1993; 20: 1892-1896.

19. Shenavandeh S, Habibi S. Nailfold capillaroscopic changes in patients with systemic lupus erythematosus: correlations with disease activity, skin manifestation and nephritis. Lupus 2017; 26: 959-966, DOI: 10.1177/0961203316686702.

20. Cutolo M, Matucci Cerinic M. Nailfold capillaroscopy and classification criteria for systemic sclerosis. Clin Exp Rheumatol 2007; 25: 663-665.

21. Jakhar D, Grover C, Singal A, Das GK. Nailfold capillaroscopy and retinal findings in patients with systemic sclerosis: is there an association? Indian Dermatol Online J 2020; 11: 382-386, DOI: 10.4103/idoj.IDOJ 26419.

22. Szucs G, Szekanecz Z, Aszalos Z, et al. A wide spectrum of ocular manifestations signify patients with systemic sclerosis. Ocul Immunol Inflamm 2019: 1-9, DOI: 10.1080/09273948.2019. 1657467 [Online ahead of print].

23. Aissopou EK, Bournia VK, Protogerou AD, et al. Intact calibers of retinal vessels in patients with systemic sclerosis. J Rheumatol 2015; 42: 608-613, DOI: 10.3899/jrheum.141425.

24. Wigley FM, Flavahan NA. Raynaud's Phenomenon. New Engl J Med 2016; 375: 556-565 DOI: 10.1056/NEJMra1507638.

25. Shenavandeh S, Hashemi SB, Masoudi M, et al. Hearing loss in patients with scleroderma: associations with clinical manifestations and capillaroscopy. Clin Rheumatol 2018; 37: 24392446, DOI: 10.1007/s10067-018-4162-7. 